\title{
Krebs und Ernährung - ein Paradigmenwechsel
}

\author{
Cancer and Nutrition - a Paradigma Shift
}

Autoren

Institute
R. Imoberdorf ${ }^{1}$, M. Rühlin², P. E. Ballmer ${ }^{1}$

${ }^{1}$ Klinik für Innere Medizin, Departement Medizin, Kantonsspital Winterthur, Schweiz

${ }^{2}$ Ernährungstherapie, Departement Medizin, Kantonsspital Winterthur, Schweiz
Schlüsselwörter

- Krebsstoffwechsel

- Kohlenhydrate

- Fett

- Mangelernährung

Keywords

- cancer metabolism

- carbohydrates

- fat

- malnutrition

\section{Bibliografie}

Dol http://dx.doi.org/ 10.1055/s-0034-1387665

Aktuel Ernahrungsmed 2015;

40: $143-146$

(c) Georg Thieme Verlag KG

Stuttgart · New York

ISSN 0341-0501

Korrespondenzadresse

\section{Dr. med. Reinhard Imoberdorf}

Klinik für Innere Medizin,

Departement Medizin,

Kantonsspital Winterthur

8401 Winterthur

Tel.: ++41 (0)52 2662121

Fax: ++41 (0)52 2664706

reinhard.imoberdorf@ksw.ch

\section{Zusammenfassung \\ $\nabla$}

Deutliche internationale Unterschiede in der Häufigkeit von Krebs haben zu der Vermutung geführt, dass die Ernährung ein wichtiger Risikofaktor für die Entstehung von Krebs sein könnte. Experten sind der Meinung, dass die Ernährung in 30-35\% mitverantwortlich für die Entstehung von bösartigen Tumoren ist. Bereits vor Tumordiagnose haben viele Patienten Gewicht verloren als Folge einer Unter- oder Mangelernährung. Häufig verschlechtert sich der Ernährungszustand während der Erkrankung weiter. Als Folge der Tumorerkrankung sowie therapiebedingt führen Appetitlosigkeit, Geschmacksveränderungen, Übelkeit, Erbrechen zusätzlich zu Mangelund Unterernährung. Die Mangelernährung hat einen erheblichen Einfluss auf die Prognose der Erkrankung und auch auf die Verträglichkeit der Therapie. Deshalb ist die Unterstützung der stark beeinträchtigten Patienten bei der Nahrungsaufnahme eine wichtige und vordringliche ärztliche und pflegerische Tätigkeit. Die physiologischen, pathophysiologischen, genetischen und molekularbiologischen Stoffwechselprozesse bei Krebs sind hochkomplex reguliert, deuten in ihrer Gesamtheit darauf hin, dass eine fett- und eiweißbasierte Ernährung favorisiert werden sollte, was einem Paradigmenwechsel zur sog. gesunden, ausgewogenen Ernährung mit viel Früchten, Gemüse und komplexen Kohlenhydraten entspricht. Die evidenzbasierte Datenlage wird aber durchaus kontrovers diskutiert.

\section{Abstract \\ $\nabla$}

Substantial international differences in the prevalence of cancer disease suppose that nutrition may be an important factor in the development of cancer. Many experts believe, that nutritional factors may contribute up to $35 \%$ to the development of malignant tumors. Many patients have lost substantial body weight already at the time of the diagnosis of the disease as consequence of undernutrition and malnutrition, respectively. During the course of the disease the nutritional status often is deteriorating further. Caused by both the cancer disease itself and the treatment, loss of appetite, changes in taste, nausea and vomiting may additionally contribute to undernutrition. Undernutrition is a relevant factor for the outcome of the disease and for the tolerance of the treatment as well. Therefore, supporting the heavily impaired patients in nutritional intake is of paramount importance and an urgent task for physicians and nurses. In view of physiology, pathophysiology, genetics and molecular biology, metabolic processes in cancer are highly complex regulated and there is increasing evidence that a diet rich in fat and protein is favourable. This, however, implies a paradigma shift away from the "healthy" balanced diet rich in fruit, vegetable and complex carbohydrates. So far, the evidence based data of this new concept is, however, a controversial issue.

\section{Einleitung \\ $\nabla$}

Schätzungen gehen davon aus, dass 30-35\% der Krebserkrankungen mit der individuellen Ernährungsweise verbunden sind [1, 2]. Der Einfluss der Ernährungs- und Lebensweise auf die Entstehung von Krebs wird eindrucksvoll illustriert, wenn man die Häufigkeit und Verteilung von Krebserkrankungen weltweit betrachtet (http:// globocan.iarc.fr/Pages/Map.aspx). Länder Osteuropas weisen mit 300-400 Fällen auf 100000 Einwohner viel höhere Krebsraten auf im Vergleich mit Kanada mit 260 Fällen auf 100000 Einwohner. In den südostasiatischen Ländern ist die 
Tab. 1 Einfluss von Ernährungs- und Lebensstilfaktoren auf die 10-Jahres-Gesamt- und Krebssterblichkeit (Ref. [5]).

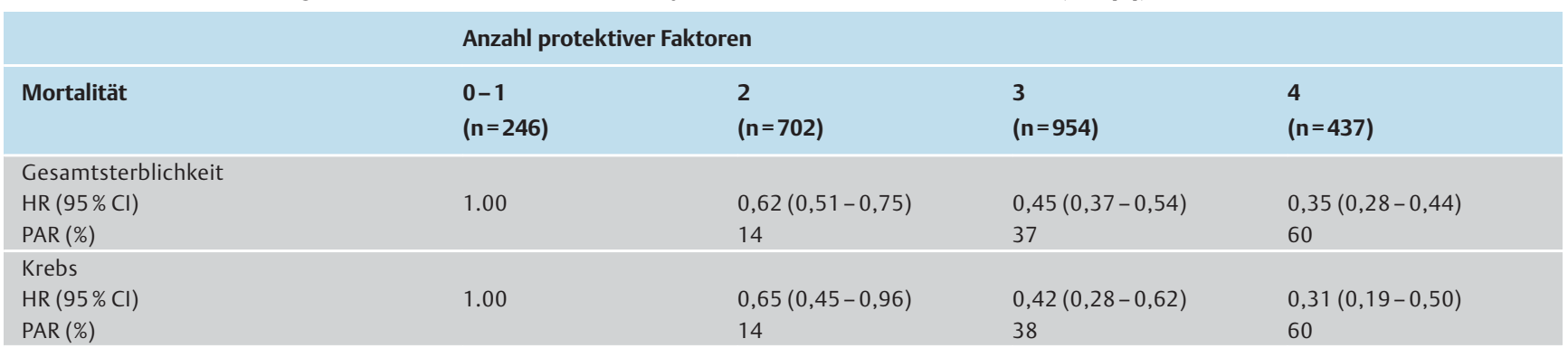

$\mathrm{HR}=$ hazards ratio; $\mathrm{Cl}=$ confidence interval; $\mathrm{PAR}=$ population-attributable risk

Zahl der Krebserkrankungen mit etwa 100 Fällen auf 100000 Einwohner ebenfalls deutlich niedriger. In diesem Zusammenhang spielen neueste Erkenntnisse der Epigenetik eine große Rolle. Nicht die Anzahl der Gene entscheidet über die Komplexität des Organismus, sondern deren Regulation und gegenseitige Beeinflussung [3]. Für die epigenetische Regulierung von entscheidender Bedeutung sind Umweltfaktoren, wie die Ernährung oder das soziale Umfeld.

Die exakten Krebsauslöser in der Nahrung sind aber häufig nicht bekannt. Die Erforschung der Ernährungsfaktoren hat sich als äußerst komplex erwiesen. Wir gehen heute davon aus, dass in der Entstehung der Krebserkrankungen eher das Zusammenspiel einer Reihe von Inhaltsstoffen eine Rolle spielt als isolierte Einzelsubstanzen. So führt eine mediterrane Ernährung nachweislich zu einer signifikanten Senkung der Gesamtsterblichkeit, der Herzinfarkt- und Krebssterblichkeit [4, 5]. Bei manifester Krebserkrankung mit konsekutivem Gewichtsverlust müssen diese gesunden Ernährungsmuster jedoch relativiert werden. Viele Krebspatienten weisen zum Zeitpunkt der Diagnose eine Mangelernährung auf. Diese führt zu einer Abnahme der Lebensqualität, der Leistungsfähigkeit und zu einer schlechteren Prognose [6].

\section{Krebs - Prävention versus Therapie \\ $\nabla$}

Im HALE-Projekt (The Healthy Ageing: a Longitudinal Study in Europe) wurden Daten aus 2 europäischen Kohortenstudien (SENECA und FINE) zusammengefasst [5]). Die Kohorten umfassten 2339 Frauen und Männer im Alter von 70-90 Jahren. Untersucht wurde, wie sich eine Mittelmeerdiät, Nichtrauchen, mäßiger Alkoholgenuss und mindestens $30 \mathrm{~min}$ Bewegung pro Tag auf die Überlebensrate auswirkte. Während der Beobachtungszeit von 10 Jahren starben 935 Personen, davon 371 an Herz-Kreislauf-Erkrankungen und 233 an Krebs. Mit einer signifikant niedrigeren Mortalität assoziiert waren Essen einer Mittelmeerdiät (Hazard Ratio [HR] 0,77; 95\% confidence intervall [CI] 0,680,88), mäßiger Alkoholkonsum (HR 0,78; CI 0,67-0,91), körperliche Aktivität (HR 0,63; CI 0,55-0,72) und Nichtrauchen (HR 0,65; CI 0,57-0,75). Hier interessiert die Frage, wie viele Krebserkrankungen vermeidbar sind, wenn diese 4 ,Low-risk“-Faktoren (Mittelmeerdiät, mäßiger Alkoholkonsum, körperliche Aktivität, Nichtrauchen) befolgt werden. Wenn alle 4 „Low-risk“-Faktoren erfüllt waren, konnten $60 \%$ der Krebsfälle verhindert werden (॰ Tab. 1).

Die im Laufe der Evolution selektierten Nahrungsmittel enthalten gesundheitsfördernde Wirkstoffe mit krebshemmenden Eigenschaften. Die sekundären Pflanzeninhaltsstoffe, z.B. Poly- phenole, besitzen hochwirksame krebshemmende Eigenschaften, die in die Prozesse eingreifen, welche an der Entwicklung von Krebs beteiligt sind ( $\bullet$ Tab. 2, mod. nach [7]). Es besteht deshalb kein Zweifel, dass sich mediterrane Lebensstil- und Ernährungsmuster positiv auf die Gesundheit auswirken können.

Bereits vor Tumordiagnose haben viele Patienten Gewicht verloren. Häufig verschlechtert sich der Ernährungszustand während der Erkrankung weiter. Als Folge der Tumorerkrankung sowie therapiebedingt führen Appetitlosigkeit, Geschmacksveränderungen, Übelkeit, Erbrechen zusätzlich zu Mangelernährung, welche einen erheblichen Einfluss auf die Prognose und auch auf die Verträglichkeit der Therapie hat [6]. In dieser Situation hat die Optimierung der Kalorien- und Eiweißzufuhr hohe Priorität. In der Praxis bedeutet das Zufuhr von mehr Fett und Eiweiß gegenüber Kohlenhydraten. Dies entspricht einem Paradigmenwechsel zur propagierten „gesunden Ernährung“, die einen Anteil von mindestens $50 \%$ Kohlenhydraten gemessen an der Gesamtkalorienzahl fordert. Experimentell gibt es verschiedene Hinweise, dass eine fett- und eiweißreiche Ernährung, auch unabhängig von einer Mangelernährung, für Krebspatienten von Vorteil sein könnte. Dies möchten wir im Folgenden diskutieren im Bewusstsein, dass die physiologischen, pathophysiologischen, genetischen und molekularbiologischen Prozesse hochkomplex reguliert sind und sicher zum Teil deswegen die evidenzbasierte Datenlage kontrovers ist.

\section{Krebsstoffwechsel - Von der Warburg-Hypothese zum Metabolom \\ $\nabla$}

\section{Tumorzellen brauchen Glukose für die Bildung von Lipiden}

Verschiedene Studien haben gezeigt, dass der Energiestoffwechsel bei Sarkomen und Karzinomen vorwiegend auf der Metabolisierung von Glukose beruht, während Fettsäuren und Ketonkörper von untergeordneter Bedeutung sind [8-10]. Epidemiologische Studien zeigen einen Zusammenhang zwischen hohem glykämischem Index und vermehrter Prävalenz von malignen Erkrankungen (in [11]). Zudem ist auf biochemischer Ebene der zentrale Abbauweg der Glukose, die Glykolyse, seit vielen Jahrzehnten mit der Wachstumsrate von malignen Zellen in Verbindung gebracht worden. In der Glykolyse kann die Glukose in Abwesenheit von Sauerstoff (anaerob) metabolisiert werden, im Gegensatz zur oxidativen Phosphorylierung über die Atmungskette (zelluläre Respiration) in den Mitochondrien. 
Tab.2 Wirkmechanismen krebshemmender sekundärer Pflanzeninhaltsstoffe (mod. nach Ref. [7]).

\begin{tabular}{|lllllll} 
& Grüntee & Kurkuma & Knoblauch & Beeren & Tomaten & n-3-FS \\
\hline Hemmung der Vermehrung von Tumorzellen & $\mathbf{X}$ & $\mathbf{X}$ & $\mathbf{X}$ & $\mathbf{X}$ & $\mathbf{X}$ & $\mathbf{X}$ \\
\hline Induktion des Zelltods & & $\mathbf{X}$ & $\mathbf{X}$ & $\mathbf{X}$ & \\
\hline Störung Angiogenese & $\mathbf{X}$ & $\mathbf{X}$ & & $\mathbf{X}$ & $\mathbf{X}$ \\
\hline
\end{tabular}

n-3-FS= Omega-3-Fettsäuren

\section{Die Warburg-Hypothese}

Otto Warburg stellte schon 1924 die Hypothese auf, dass eine Steigerung der Glykolyserate bzw. eine Verminderung der zellulären Respiration pathogenetisch ursächlich für die Entstehung malignen Zellwachstums sei [12]. Normale Zellen brauchen für ihr Wachstum Lipide, welche sie selber aus Glukose bilden. Unter normalen aeroben Bedingungen werden $80 \%$ der Lipide einer Zelle aus Glukose gebildet und 20\% aus Glutamin. Für Tumorzellen ist die anaerobe Glykolyse ein evolutionärer Vorteil. In fortgeschrittenen Stadien kann die arterielle Versorgung nicht mit dem Tumorwachstum Schritt halten. Dies führt zu hypoxischen $\mathrm{Zu}-$ ständen, wobei nun die Tumorzelle über die anaerobe Glykolyse Glukose für die Bildung von Lipiden verwendet und somit trotz ungünstigen Bedingungen weiter proliferieren kann. Spezifische Veränderungen des intrazellulären Glukosestoffwechsels können also in kausalem Zusammenhang stehen mit der unkontrollierten Proliferation von Zellen.

\section{Ernährung für Tumorpatienten - Kohlenhydratarm, fett- und eiweißreich?}

Basierend auf der Warburg-Hypothese stellt sich die Frage, ob kohlenhydratarme, ketogene Ernährungsformen zu einer Reduktion der Tumorzellproliferation führen können. Die tierexperimentellen Daten zu dieser Hypothese sind sehr kontrovers. Bei Mäusen mit Tumoren kam es nach initialer Wachstumsverzögerung bei den ketogen ernährten Tieren nach 20 Tagen zu einer Beschleunigung des Tumorwachstums. In einem anderen Experiment kam es zwar zu einer Tumorrückbildung, jedoch zum Überleben von Tumorstammzellen, welche Ketonkörper und Laktat zur Energiegewinnung nutzen können (in [13]).

Bei einem Patienten mit einem Rezidiv eines Desmoidtumors wurde eine lipidreiche total parenterale Ernährung mit $28 \mathrm{kcal}$ Fett $/ \mathrm{kg} \mathrm{KG} /$ Tag und $1,5 \mathrm{~g}$ Aminosäuren $/ \mathrm{kg} /$ Tag verabreicht. Lediglich $40 \mathrm{~g}$ Glukose/Tag waren erlaubt, zudem wurde die Glukoneogenese durch orale Zufuhr von Hydrazinsulfat gehemmt. Die Ernährung wurde gut toleriert und die Tumorgröße veränderte sich nicht wesentlich, aber eine messbare Läsion auf dem Rücken bildete sich leicht zurück [14].

Klinische kontrollierte Studien liegen bisher nicht vor. Ob alle Krebspatienten kohlenhydratarm und somit fett- und eiweißreich ernährt werden sollten, lässt sich zurzeit nicht abschliessend beurteilen.

\section{Tumorzellen brauchen nicht nur Glukose}

Wie schon erwähnt, leben Tumorzellen in einer unwirtlichen Umgebung. Es fehlt an Sauerstoff und Glukose. Neueste Studien konnten zeigen, dass Tumorzellen neben der anaeroben Glykolyse die für die Proliferation notwendigen Lipide über alternative Stoffwechselwege bilden können, nämlich über die Metabolisierung von Glutamin [15]. Überraschend war, dass Tumorzellen diesen alternativen Stoffwechselweg viel häufiger nutzten als man es sich bisher vorgestellt hatte. Wie oben erwähnt, werden unter normalen aeroben Bedingungen 80\% der Lipide einer Zelle aus Glukose gebildet und 20\% aus Glutamin. Unter Hypoxiebedingungen der Tumorzelle kehrt sich dieses Verhältnis um, 80\% der notwendigen Lipide kann die Tumorzelle aus Glutamin gewinnen!

In der onkologischen Diagnostik und Therapie sind genetische Veränderungen fester Bestandteil des klinischen Alltags. Die aktuelle intensive Forschung ist daran, die Krebskrankheiten umfassend über die Entschlüsselung des sog. Metaboloms zu charakterisieren. Als Metabolom werden, angelehnt an die Begriffe Genom und Proteom, die spezifischen Eigenschaften eines zellulären Stoffwechsels zusammengefasst. Mittels „high-resolution magic angle spinning nuclear magnetic resonance spectroscopy“ konnte in einer Studie bei 44 Patienten mit kolorektalen Tumoren sicher zwischen gesunder Kolonschleimhaut, Kolonkarzinom und Rektumkarzinom unterschieden werden [16]. Krebsgewebe zeichnete sich durch signifikant höhere Konzentrationen von Laktat, Taurin und Isoglutamin und tiefere Konzentrationen von Lipiden und Triglyzeriden im Verhältnis zu gesunder Darmschleimhaut aus. Gegenüber Proben von Rektumkarzinomen wiesen die Proben von Kolonkarzinomen signifikant höhere Konzentrationen von Azetat und Arginin und tiefere Konzentrationen von Laktat auf. Gesunde Mukosa konnte mit diesen Parametern sicher von maligner Mukosa unterschieden werden. Die Bestimmung des Metaboloms stellt eine direkte Momentaufnahme der zellulären Vorgänge dar, das Gewebe muss nicht denaturiert werden, kann also für weitere histologische Aufarbeitung verwendet werden und das Resultat ist innerhalb von 30 min verfügbar.

Ob solche Stoffwechselprofile durch eine spezifische Ernährung veränderbar sind, lässt sich nur spekulieren. Theoretisch betrachtet, würden die oben beschriebenen Befunde eine Ernährung auf Basis von Fett und Eiweiß mit Einschränkung der Kohlenhydrate favorisieren. Diese Annahme wird durch eine kürzlich publizierte prospektive Beobachtungsstudie bei 1011 Patienten mit Kolonkarzinom Stadium III unterstützt [17]. Höhere glykämische Last und höhere gesamte Kohlenhydrataufnahme (Vergleich zwischen höchster und tiefster Quintile) waren signifikant mit erhöhtem Risiko für ein Rezidiv und für Gesamtsterblichkeit assoziiert.

\section{Klinischer Alltag - Krebs bedeutet häufig Mangel- ernährung}

In der Deutschen Studie zur Mangelernährung im Krankenhaus [18] wurde bei $27,4 \%$ der Patienten nach dem SGA (Subjective Global Assessment) eine Mangelernährung diagnostiziert, wobei $43 \%$ der Patienten im Alter von über 70 Jahren betroffen waren im Vergleich zu 7,8\% im Alter unter 30 Jahren. Die höchste Prävalenz der Mangelernährung wurde in geriatrischen (56,2\%), onkologischen (37,6\%) und gastroenterologischen (32,6\%) Abteilungen beobachtet. Die multivariate Analyse dieser wichtigen Studie ergab 3 unabhängige Risikofaktoren für eine Mangelernährung: 
hohes Alter, Anzahl eingenommener (bzw. verordneter) Medikamente und maligne Erkrankungen. In einer eigenen Untersuchung bei insgesamt 32837 Patienten fanden wir bei 5978 (18,2\%) eine Mangelernährung beim Eintritt ins Krankenhaus [19]. Die Prävalenz bei den Krebspatienten betrug fast $40 \%$. Bei 3576 Patienten wurde eine orale Ernährung durchgeführt, entweder mit normaler Krankenhauskost ( $n=2018)$, oder Anreicherung der Speisen $(n=295)$ oder Zusatztrinknahrung $(n=1025)$; 238 Patienten erhielten sowohl Anreicherungen als auch Zusatztrinknahrung. Eine künstliche Ernährung wurde bei 599 Patienten durchgeführt: enteral via Sonde bei 438 Patienten, parenteral bei 116 und kombiniert enteral und parenteral bei 43 .

$50 \%$ der Patienten konnten demzufolge ihren Energie- und Eiweißbedarf durch die normale Krankenhauskost nicht decken. Sie mussten ernährungstherapeutisch unterstützt werden, wobei Anreicherung, Zusatztrinknahrung, enterale Sondenernährung und parenterale Ernährung zur Anwendung kamen.

Ein Großteil der Krebspatienten wird ambulant betreut und behandelt. Viele dieser Patienten entwickeln im Verlauf eine Mangelernährung. Im Krankenhaus der Autoren arbeitet eine Ernährungstherapeutin ständig im onkologischen Ambulatorium, sodass die nicht adäquat ernährten Patienten frühzeitig erfasst werden können. Wir haben bei 58 ambulanten Krebspatienten mit verschiedenen Karzinomen und bestehender Mangelernährung oder hohem Risiko für eine Mangelernährung eine prospektive Ernährungsstudie durchgeführt [20]. Eine individualisierte Ernährungsintervention (Beratung durch eine Ernährungstherapeutin, Anreicherung der Speisen, Trinknahrung) führte im Vergleich zur „Usual care“-Gruppe zu einer signifikanten Zunahme von $379 \mathrm{kcal}$ und 10,4g Eiweiß/Tag. Die gesteigerte Zufuhr hatte aber keinen Einfluss auf die körperliche Funktionstüchtigkeit und auch nicht auf die Lebensqualität, ein Umstand, der erst kürzlich in einer Studie bestätigt wurde [21]. Unsere Patienten hatten schon eine fortgeschrittene Krebserkrankung und die Mehrzahl der Patienten befand sich in einem schlechten Ernährungszustand. Wahrscheinlich kam die Ernährungsintervention zu spät und konnte als alleinige Maßnahme die Lebensqualität deshalb nicht mehr verbessern.

\section{Schlussbemerkungen}

Krebserkrankungen sind sehr häufig und diese Krankheiten führen im Verlauf häufig zu Mangelernährung mit markantem Gewichstsverlust. Dadurch wird die Prognose verschlechtert und die Lebensqualität sinkt. Die körperliche Funktionalität hängt von einer intakten Muskelmasse ab, was sich zusammen mit vielen anderen Faktoren auf die Lebensqualität auswirkt. Muskelschwund („muscle wasting“) ist assoziiert mit reduzierter körperlicher Leistungsfähigkeit und Immunkompetenz, erhöhter behandlungsinduzierter Toxizität und kürzerem Überleben [22]. Krebspatienten haben aber das Potenzial zur Eiweißsynthese [23]. Spezifische Aminosäuren, vor allem Leucin, können die Eiweißsynthese stimulieren, wenn andere essenzielle Aminosäuren in genügender Menge vorhanden sind. Einen optimalen Eiweißanabolismus erreicht man durch Zufuhr von hohen Dosen Leucin zusammen mit qualitativ hochwertigem Eiweiß, kombiniert mit Omega-3-Fettsäuren als Teil eines multimodalen Therapiekonzepts. Dabei sollten die antitumoralen medikamentösen und Strahlentherapien und die operativen Verfahren ergänzt werden durch ein körperliches Aktivitätsprogramm und eine frühzeitige Ernährungstherapie, welche eher fett- und eiweißbasiert sein soll und somit, wie im Titel erwähnt, einem Paradigmenwechsel zur sog. gesunden ausgewogenen kohlenhdydratliberalen Ernährung gleichkommt.

\section{Interessenkonflikt}

$\nabla$

Alle drei Autoren erhielten Referentenhonorare von Firmen der Ernährungsindustrie.

\section{Literatur}

1 Doll R, Peto R. The causes of cancer: Quantitative estimates of avoidable risks of cancer in the United States today. J Natl Cancer Inst 1981; 66: $1192-1308$

2 Doll $R$. The lessons of life: keynote address to the nutrition and cancer conference. Cancer Res 1992; 52: 2024s-2029s

3 Spork P. Das Genom und seine Regisseure. Grundlagen der Genetik und Epigenetik. Aktuel Ernahrungsmed 2013; 38 (Suppl. 01): S2 -S6

4 Trichopoulou A, Costacou T, Bamia Ch et al. Adherence to a mediterranean diet and survival in a Greek population. N Engl J Med 2003; 348: $2599-2608$

5 Knoops KTB, de Groot LCPGM, Kromhout D et al. Mediterranean diet, lifestyle factors, and 10-year mortality in elderly European men and women. The HALE project. JAMA 2004; 292: 1433-1439

6 Arends J. Ernährung von Tumorpatienten. Aktuel Ernahrungsmed 2012; 37: 91 - 106

7 Béliveau R, Gingras D. Krebszellen mögen keine Himbeeren. 12. Auflage. München: Goldmann; 2010: 978-3-442-17126-2

8 Norton JA, Burt ME, Brennan MF. In vivo utilization of substrate by human sarcoma-bearing limbs. Cancer 1980; 45: 2934-2939

9 Hagmüller E, Kollmar HB, Günther HJ et al. Protein metabolism in human colon carcinomas: in vivo investigation using a modified tracer technique with 1-(1-13C)leucine. Cancer Res 1995; 55: 1160-1167

10 Holm E, Hagmüller E, Staedt $U$ et al. Substrate balances across colonic carcinomas in humans. Cancer Res 1995; 55: 1373-1378

11 Ristow M. Glukosestoffwechsel und Tumorwachstum. Onkologe 2008; 14: $22-30$

12 Warburg O, Posener K, Negelein E. Über den Stoffwechsel der Tumoren. Biochem Z 1924; 152: 319-344

13 Hübner J, Löser C, Stoll C. Vorstellungen zur Therapie von Malignomen mit Krebsdiäten. Empfehlungen für die ärztliche Beratung. Onkologe 2013; 19: $108-116$

14 Bozzetti F, Cozzaglio L, Gavazzi C et al. Total nutritional manipulation in humans: a report of a cancer patient. Clin Nutr 1996; 15: 207-209

15 Metallo C, Gameiro P, Bell E et al. Reductive glutamine metabolism by IDH1 mediates lipogenesis under hypoxia. Nature 2012; 481: 380 384

16 Mirnezami R, Jiménez N, Li JV et al. Rapid diagnosis and staging of colorectal cancer via high-resolution magic angle spinning nuclear magnetic resonance (HR-MAS NMR) spectroscopy of intact tissue biopsies. Ann Surg 2014; 259: 1138-1149

17 Meyerhardt JA, Sato K, Niedzwiecki $D$ et al. Dietary glycemic load and cancer recurrence and survival in patients with stage III colon cancer: findings from CALGB 89803. J Natl Cancer Inst 2012; 104: 1702 - 1711

18 Pirlich M, Schütz T, Norman K et al. The German hospital malnutrition study. Clin Nutr 2006; 25: $563-572$

19 Imoberdorf $R$, Meier $R$, Krebs $P$ et al. Prevalence of undernutrition on admission to Swiss hospitals. Clin Nutr 2010; 29: 38-41

20 Uster A, Rüfenacht $U$, Rühlin $M$ et al. Influence of a nutritional intervention on dietary intake and quality of life in cancer patients: a randomized controlled trial. Nutrition 2013; 29: 1342 - 1349

21 Poulsen G, Pedersen L, Østerlind $K$ et al. Randomized trial of the effects of individual nutritional counselling in cancer patients. Clin Nutr 2014; 33: 749-753

22 Fearon K, Strasser F, Anker SD et al. Definition and classification of cancer cachexia: an international consensus. Lancet Oncol 2011; 12: 489 495

23 Chevalier S, Winter A. Do patients with advanced cancer have any potential for protein anabolism in response do amino acid therapy? Curr Opin Clin Nutr Metab Care 2014; 17: 213-218 